\title{
Optimizing the memory bandwidth with loop morphing
}

\author{
J.I. Gomez \\ DACYA UCM \\ jigomez@dacya.ucm.es \\ IMEC/ESAT KULeuven \\ S. Verdoorlaege \\ ESAT KULeuven \\ marchal@imec.be_skimo@kotnet.be \\ L. Pinuel \\ DACYA UCM \\ lpinuel@dacya.ucm.es \\ F. Catthoor \\ IMEC/ESAT KULeuven \\ catthoor@imec.be
}

\begin{abstract}
The memory bandwidth largely determines the performance of embedded systems. However, very often compilers ignore the actual behavior of the memory architecture, causing large performance loss. To better utilize the memory bandwidth, several researchers have introduced instruction scheduling/data assignment techniques. Because they only optimize the bandwidth inside each basic block, they often fail to use all available bandwidth. Loop fusion is an interesting alternative to more globally optimize the memory access schedule. By fusing loops we increase the number of independent memory operations inside each basic block. The compiler can then better exploit the available bandwidth and increase the system's performance. However, existing fusion techniques can only combine loops with a conformable header. To overcome this limitation we present loop morphing: we combine fusion with strip mining and loop splitting. We also introduce a technique to steer loop morphing such that we find a compact memory access schedule. Experimental results show that with our approach we can decrease the execution time up to $38 \%$.
\end{abstract}

\section{Introduction}

Two complementary techniques are often used to improve the memory bandwidth. On the one hand designers exploit a hierarchy of memory layers to hide the latency of the slow memories. On the other hand, they use multiple memories on each layer to increase the bandwidth.

In this paper, we focus on how to optimize the memory bandwidth from the memory layer closest to the processor. It contains in itself multiple memories. Nowadays the compiler determines how many and which memory access operations are scheduled in parallel. In the simplest case, it schedules as many memory operations in parallel as load/store units exist on the processor. It even schedules memory operations in parallel of which the corresponding data resides in the same memory. At run-time, the memory interface has to serialize these operations and stall the processor. These stalls can be avoided with multiport memories, but this comes at the cost of extra energy. Another way to execute more operations in parallel is to use multiple single-port memories and to carefully distribute the data across these memories [17]. The compiler can also optimize the instruction schedule to avoid simultaneous accesses to the same memory [20]. Unfortunately, even with this technique the dependencies between the memory accesses prevent the compiler to fill all 
available access slots. An important reason is that it schedules the operations within the scope of a basic(hyper)-block. This severely limits the scheduling freedom. If we optimize the memory bandwidth across the boundaries of a basic block, we can obtain a higher performance.

A well known global transformation is loop fusion. By combining two loop bodies, it increases the number of independent instructions in each basic block. The compiler can then better utilize the memory bandwidth. So far, loop fusion has been mainly used to increase locality and reduce the life-time of arrays (see Sect. 2). As a result, their criteria to decide which loops to combine are different from ours. Also, they only combine loops with conformable loop headers, i.e. nested loops which execute the same number of iterations. In practice, this limits the applicability of fusion.

We propose in this paper a technique to fuse unconformable loops called loop morphing, which overlaps as many iterations as possible of a loop pair. Loop morphing is a combination of loop fusion with strip mining and loop splitting. By iteratively applying loop morphing we can significantly improve the memory bandwidth utilization. We provide a heuristic to decide in which order to morph loops. Loop morphing can in principle also be used to further increase the instruction level parallelism inside basic blocks [15]. We have implemented our technique within SUIF and automatically generate transformed C-code. We have measured on a VLIW (Trimaran) simulator performance improvements up to $38 \%$.

This paper is organized as follows. First, we discuss the related work (Sect. 2). Then, we motivate with an example why loop morphing is needed (Sect. 3). Thereafter, we explain our approach (Sect. 5-4) and finally, we validate it with several examples (Sect. 6).

\section{Related Work}

Memory optimization is a widely researched topic (see [1] and [14] for good overviews). Several techniques have been introduced to optimize the memory bandwidth. These techniques most often integrate memory bandwidth optimization within the compiler/linker $[17][12][5]$. In [17] the performance is maximized by distributing the data across the different memories such that as many accesses as possible can be executed in parallel. The authors of [12] and [5] propose an instruction scheduling technique. They expose the access latency of SDRAM memories to the compiler. The scheduling is done after the data has been assigned to the memories. [20] optimizes the memory bandwidth in a separate step before compilation. They optimize the schedule of the memory operations and the data assignment together. They output a (partial) data assignment which constrains the final instruction scheduling. It guarantees that enough memory bandwidth exists to meet the deadline, while remaining as energy-efficient as possible. Above techniques optimize the memory bandwidth within the boundaries of a basic block. This limits the performance if not enough independent memory operations exist in each basic block.

More global optimization techniques can further improve the performance. In the past, several authors have proposed techniques to globally schedule instructions to parallelize code $[9][8][10]$. They do not focus on how to optimize the memory bandwidth. [19] defines a global operation schedule which reduces the number of memory ports. However, it does not take into account which data structures are accessed or how they are mapped in the memory. Furthermore, the resulting schedule is implemented with a dedicated controller, which is quite different from current processors.

Loop transformations are an interesting alternative to optimize the code across the boundaries of a basic block. Originally, they have been developed to increase the par- 
allelism inside tasks [11], increase regularity [4][13][3] and/or improve data locality [2]. One particular transformation, loop fusion, is often used to increase the size of a loop body. The compiler can then detect more instruction level parallelism [15]. This is also what we need for memory bandwidth optimization. However, existing techniques can only fuse loops which execute the same number of iterations. In practice, this condition limits the applicability of fusion. Several authors therefore combine loop fusion with loop shifting (see [18] for an overview), but they target a different optimization objective. They want to increase locality or the life-time of arrays. As a consequence, they only partially overlap the iteration spaces of different loops. In contrast, we want to maximally overlap iteration spaces. We optimize then the memory bandwidth in as many iterations as possible. Furthermore, the mechanism to decide which loops to combine is different. We cannot thus directly reuse these techniques in our context. We have implemented our loop morphing technique in the polyhedral mode (see also [18] for an extended overview on its related work).

\section{Motivational example}

We explain with an example why loop morphing helps to better utilize the available bandwidth. The example consists of two data-dominated loops (see code in Fig. 1-left) .

We execute them on a VLIW simulator (Trimaran) configured with two memory ports. Both ports are connected with a crossbar to two memories. Since both loops are data dominated, the schedule of the memory accesses determines the performance of the loops. We assume that the remaining operations can be performed in parallel with the memory accesses or take only limited time. We now study the influence of loop fusion on the length of the memory access schedule.

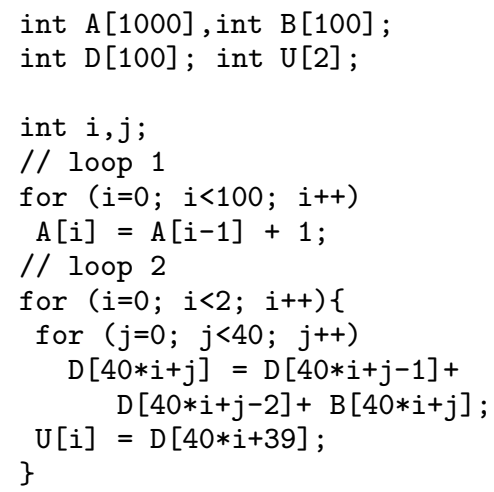

Figure 1. Motivational example: original code (left), code after loop morphing (right)

Industrial compilers are unaware of the underlying memory architecture. During instruction scheduling they simply assume that a memory operation is ready after $n$ cycles. When the compiler has scheduled two accesses to the same memory in parallel, the processor needs to stall one of the operations. The execution takes then longer than presumed. Hence a large difference often exists between the expected and the effective performance of the processor. Specifically, using an existing modulo scheduler [16], the memory access schedule of the inner-loops in our example is expected to take 360 cycles (see Fig. 2-(a)). When we simulate the code on our platform, the execution time can take up to 520 cycles when the linker stores $b$ and $d$ in the same memory. Even if both data structures are in a different memory, the execution time remains 440 cycles, because the processor still has to 
serialize the parallel accesses to $d$ in loop 2 .
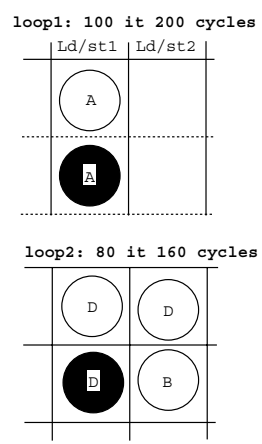

expected perf.: 360 cycles actual perf.: 440-520 cycles

(a)

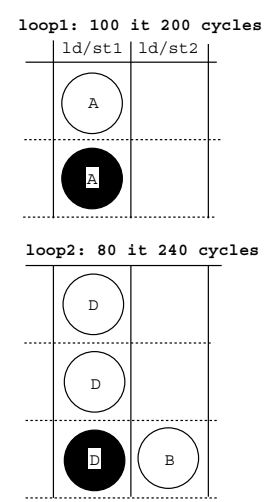

perf.: 440 cycles (b)

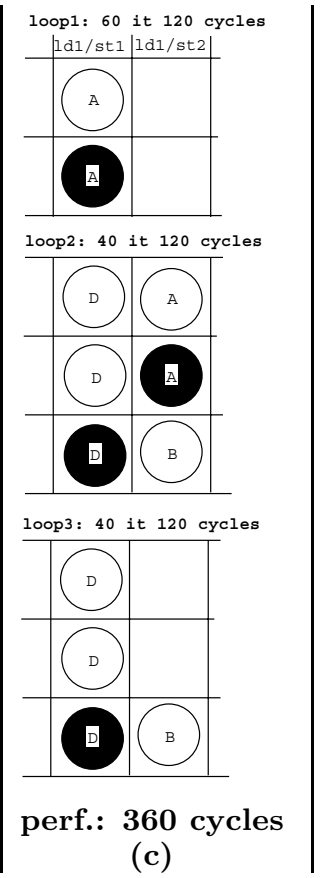

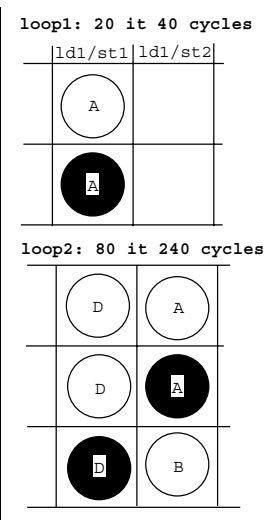

perf.: 280 cycles

(d)

Figure 2. Empty issue slots in the memory access schedule of the inner-loops: (a) existing compiler; (b) with assignment constraints; (c) existing loop fusion; (d) with loop morphing

Many memory access slots remain empty because: (1) inter-iteration dependencies; for instance the initiation interval of loop 1 is 2, because $A$ depends on itself. Hence, only $50 \%$ of the available memory slots are used; (2) resource limitations. In loop 2, the operations to $D$ cannot be scheduled in parallel since we do not use multi-port memories to save energy. As a result, the total schedule length is longer than required.

With loop fusion we can further reduce the execution time of the code. For instance, we may fuse loop 1 and loop 2. Current fusion techniques (such as applied in [15]) will not combine both loops since they have a non conformable loop header. The techniques which integrate loop fusion with loop shifting (such as [18]) can maximally overlap 40 out of 80 iterations of loop 2 (see 2-(c)). They reduce the execution time to 360 cycles. However, by first splitting loop 1 and then strip mining it, we can generate conformable loop headers. As a result, we can fuse all 80 iterations of loop 2 with loop 1 . After fusion, the execution takes only 280 cycles (see Fig. 2-(d)). We fuse as many iterations as possible of each loop, because this minimizes the total schedule length. The resulting code is shown in Fig. 1-right.

From this example, we conclude that with loop fusion we can outperform existing scheduling techniques (from 440 to 280 cycles) without altering the data assignment. However, (1) we should be able to fuse non conformable loops, (2) we should maximize the number of overlapping iterations after fusion and (3) finally, if we are able to fuse any loop pair, the number of loop pairs which we can combine becomes much larger. To obtain a good performance, we should then carefully decide which ones to combine. 


\section{Fusing two non-conformable loops: loop morphing}

As indicated in Sect. 3, if two loop nests are not conformable, current fusion techniques can only overlap few iterations. For instance, consider the two loops nests in Fig. 3. We show them together with their iteration domains. The iteration domain of a loop nest is the set of iterators for which each loop is executed. These domains can be represented by (unions of) polytopes (as those shown in Fig. 3, where each dot corresponds to a single iteration).

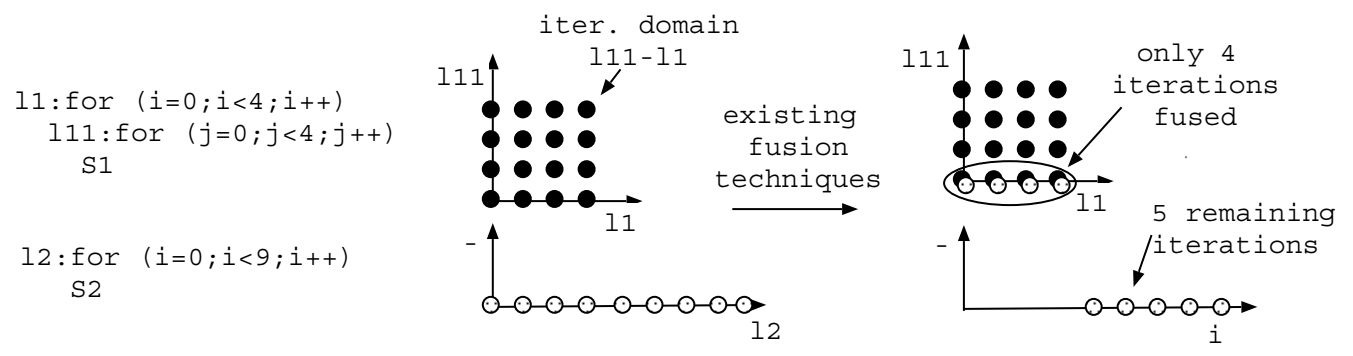

Figure 3. Existing fusion techniques can only overlap a limited number of iterations

The 11-111 loop nest is two dimensional and the 12 is one dimensional. Techniques such as proposed in [18] can only fuse four out of nine iterations. This limits the performance improvement. To maximize the number of overlapping iterations after fusion, we define the loop morphing problem: "to find sequences of loop transformations $\pi_{k}$ and $\pi_{l}$ for loop nests $k$ and $l$, such that $\operatorname{Card}\left(\pi_{k}\left(D_{k}\right) \cap \pi_{l}\left(D_{l}\right)\right)$ is maximized" (where $D_{i}$ stands for the iteration domain of loop nest $i$ ).

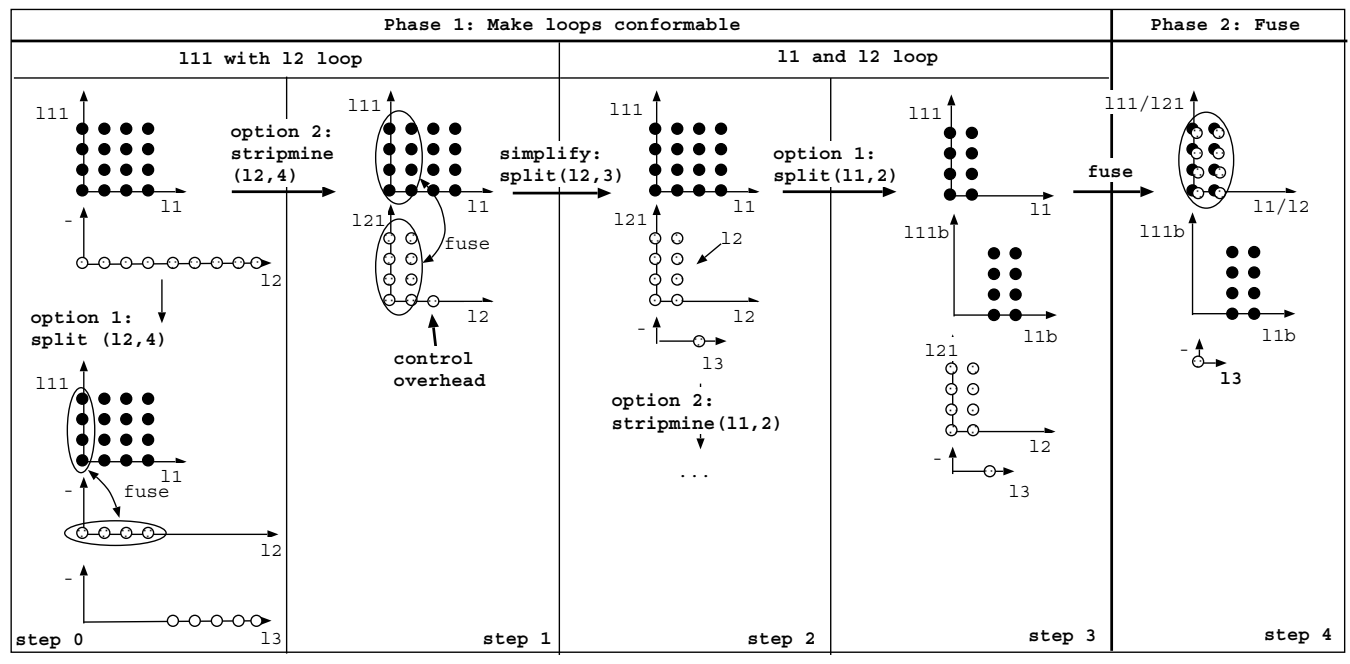

Figure 4. Morphing heuristic applied on an example

We propose a heuristic to solve this loop morphing problem (Algo. 2). We explain it with the help of the example in Fig. 3. In our algorithm we gradually make the iteration domains of the loop-nests $l 1 / l 11$ and $l 2$ more conformable. We start from the inner-most loop at which they are non-conformable (line 4: cur_lev_l1/2). For our example, this means that we start with $l 11$ and 12 . They are the inner most non-conformable loops because they execute a different number of iterations. We then make these loops conform, i.e. we 
ensure that they have the same length (line 6-10). Two options exist to make both loops conformable: we can either split or strip-mine the longest loop such that it has the same length as the shortest one (line 7/9). As explained below, we use a simple criterion to decide which transformation to apply (line 6). After each iteration, one more dimension of both iteration domains has become conformable. During the subsequent iterations of our algorithm, we reshape the next inner-most non-conformable loops (lines 3-7). We repeat this until both loop nests are conformable. At the end, we can then simply fuse (line 8) both iteration loop nests with any traditional technique [6].

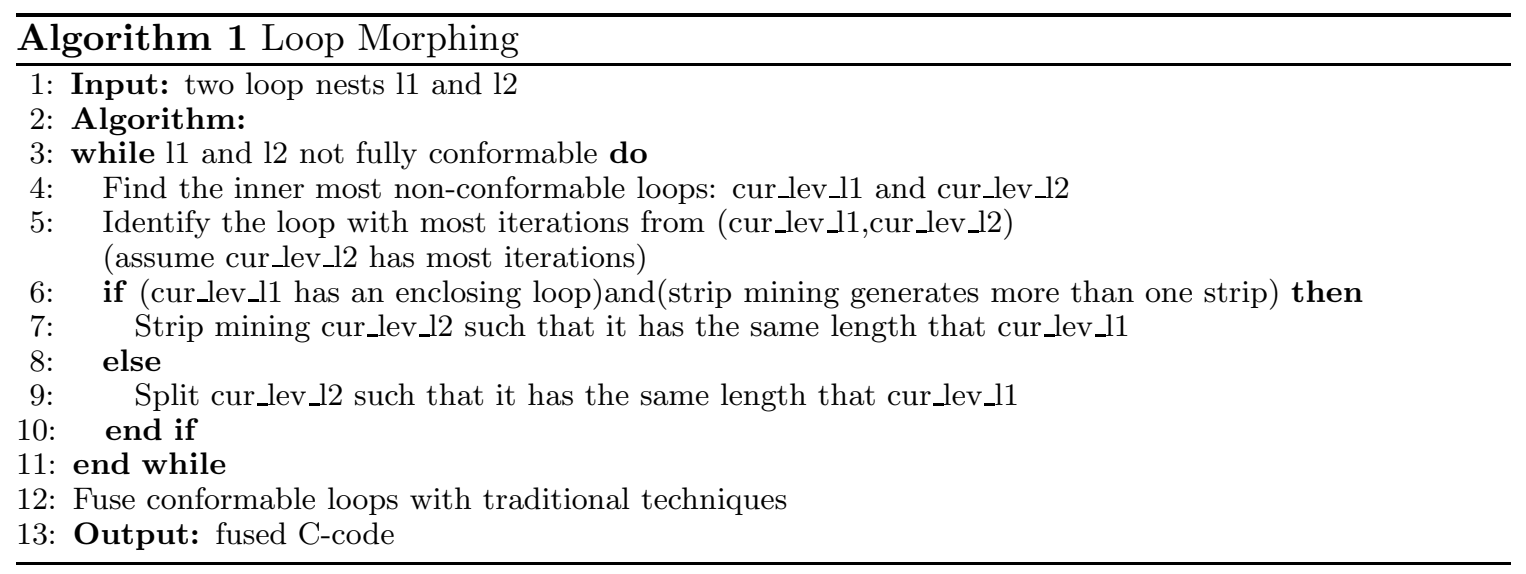

We apply this algorithm on our example (see Fig. 4). In step 0, $l 11$ and 12 are the inner most non-conformable loops. We thus either need to split or strip mine 12. Consider the case that we split 12 (option 1 in Fig. 3). The new 12 has the same number of iterations as l11. As a result, it becomes conformable with $l 11$ and we can fuse both loops. Since the new 12 only contains four out of nine iterations, the maximum number of iterations which we can overlap is limited. Splitting generates also the second loop, 13 , which contains the remaining iterations of the original 12 .

The second option is to strip mine 12 with the length of $l 11$ as strip size ${ }^{1}$ (see step 1 ). We then generate a new inner loop, 121, which has the same length as 111 . The iteration domains composed by $12 / l 21$ and $l 1 / l 11$ are more conformable. Because the iteration domain of the l2/l21 contains now $2 \times 4+1$ iterations, more iterations can be potentially fused. In general, strip mining adds an extra dimension to a loop nest. The extra dimension is only useful if it helps to better overlap both iteration domains. Assume an example which consists of two one-dimensional loops. Strip mining one of them transforms its iteration domain in a two-dimensional one, thereby making both iteration domains more different than originally. To avoid creating extra mismatch, we impose as a simple constraint that $l 11$ should have at least one more enclosing loop. If so, we can after-wards try to match the newly-generated outer loop (in casu l2) with it. Furthermore, to avoid loop control overhead, we impose as a second constraint that the newly-generated outer loop (12) contains at least two iterations. In our example, the above conditions are met. Hence, we strip mine 12 (step 1).

As a practical detail, note that the length of 12 is not a multiple of the length of the $l 11$ loop. Consequently, after strip mining a condition exists in the body of 121 . To avoid it, we move the $(12=3,121=1)$-th iteration of 12 to a separate loop, 13 (step 2).

Since both loops nests are not fully conformable yet, we repeat the above steps. We

\footnotetext{
${ }^{1}$ thus the length of the inner loop
} 
detect again the inner most dimensions which make both loops nests non conformable ( $l 1$ and 12 ). Thereafter, we transform the longest loop $l 1$ such that it has the same length as $l 2$. Like before, two options exist, we can either apply strip mining or index splitting. However, because 12 has no enclosing loop any more, strip mining here creates more mismatch. Hence, we simply split the $l 1$ loop using the length of $l 2$ loop (step 3). Thereafter, both loop nests $l 1 / l 11$ and $l 2 / l 21$ are conformable and we can fuse both loop nests (step 4).

With loop morphing, we can combine any pair of loop nests with manifest loop bounds. It can be used for many purposes such as increasing the ILP inside basic blocks (e.g. [15]). In this paper, we have applied it to optimize the memory bandwidth. In the next section, we outline a technique to iteratively steer morphing to optimize the memory bandwidth.

\section{$5 \quad$ Greedy Iterative Morphing}

In applications, usually several loops nests exist which can be fused. To maximize the performance, we pairwise fuse loops until no pair remains which reduces the execution time after fusion. We explain a heuristic to decide which loops to combine (see Algo. 2). The input of our algorithm is the initial C-code of the application (line 1.1). We also take into account how the data is stored inside the memories. The data assignment determines which basic blocks have many empty memory access slots. These basic blocks are typically interesting candidates for fusion. The assignment constrains are modeled with a conflict graph (line 1.2). The nodes correspond to the data structures. An edge between two nodes indicates that their data is stored in different memories. From a given conflict graph we can usually derive several valid data to memory assignments. Hence, it only partially constraints the assignment (see also [20]). Finally, we input also a description of the memory architecture. We use this description while estimating the memory access schedule length of a loop (line 1.3).

Our algorithm first parses the C-code to generate our internal model (line 3). Then, we enumerate all possible fusion candidates (line 4). We represent them with a fusion graph (FG) (similar to [7]). The nodes of the graph correspond to the loops in the code. An edge between a pair of loops marks fusible loops. We conservatively assume that loops are only fusible when no (direct/or indirect) dependencies exist between them. Furthermore, if we fuse two loops, the number of data structures which we need to store on the local memories also increases. Therefore, in each iteration the required memory size should not exceed the size provided by the architecture.

The edges are annotated with the fusion gain. The fusion gain is an estimation of the relative system's performance gains after fusion (line 5 ). We estimate the initial application performance $\left(t_{\mathcal{L}}\right)$ as the sum of the schedule length of each basic block in the code (line 5.1). In case the basic block can be software-pipelined, the schedule length of each basic block is the product of its minimum initiation interval (MinII [16]) with its number of iterations $\left(i_{k}\right)$. We obtain MinII with a modulo scheduler [16] which takes the number of memory ports and the access latency into account. We only schedule the background memory operations and thus omit all other instructions. This simplifies the performance estimation, but remains sufficiently accurate because we focus on data dominated applications. We also take the assignment constraints into account during scheduling. Particularly, we only schedule memory operations in parallel if a conflict exists between their corresponding data in the conflict graph. After computing the initial performance, we compute for each pair of fusible loops the performance gains after fusion (5.2). It is the product of the fusion gain per iteration times the number of fused iterations. The fusion gain per iteration is the 


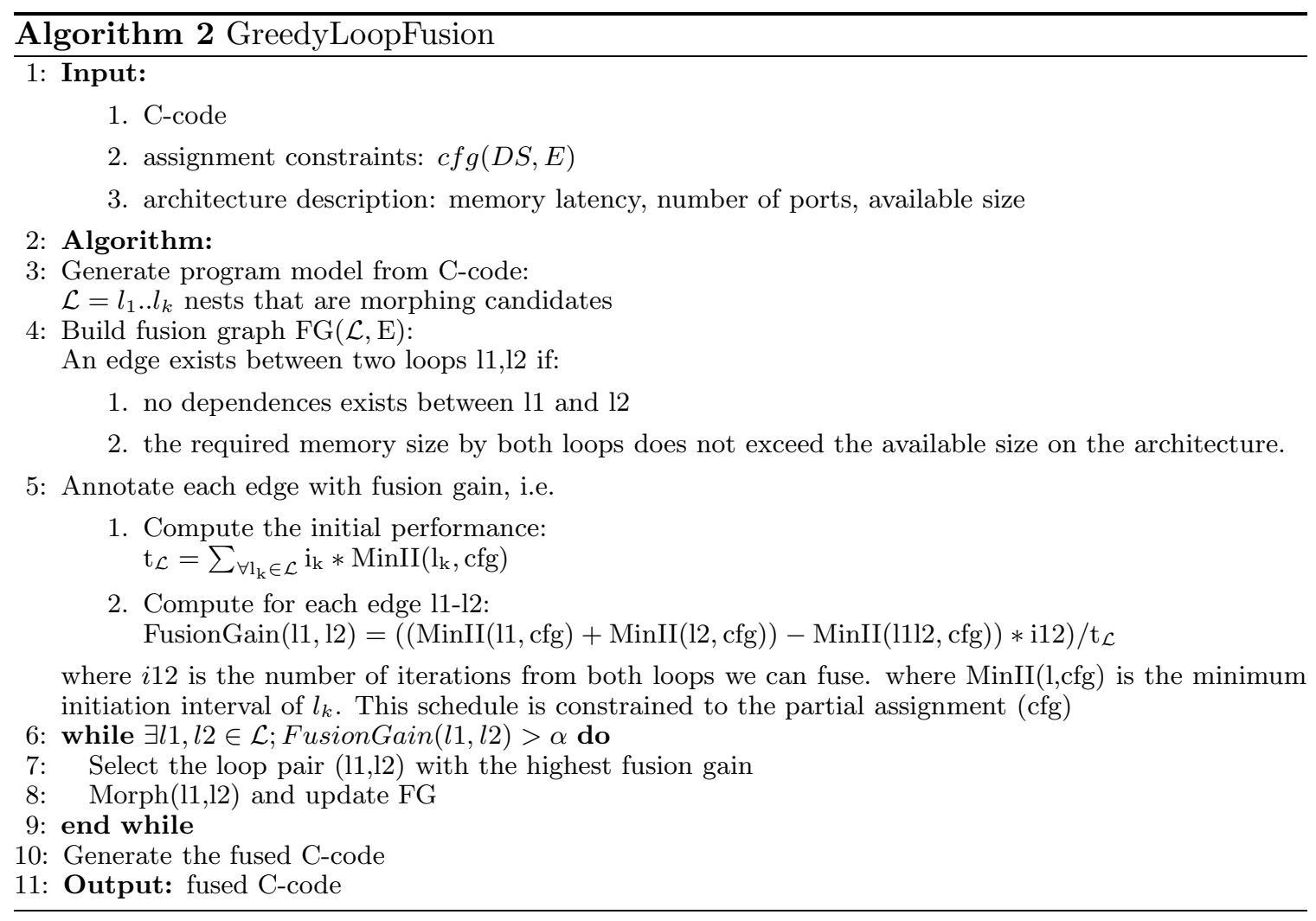

difference of the initiation interval of the fused length with the sum of those of the original loops. By executing our morphing heuristic we can estimate $i_{12}$, i.e. how many iterations of two loop nests can be overlapped.

During each iteration of the while-loop, we fuse the loop pair with the highest fusion gain (line 7). An extra condition is that the fusion gain should be larger than a threshold $\alpha$. In this way, we prevent fusing loops which have no impact on the performance, but would only generate extra control overhead. We use loop morphing to fuse the loops. Although this technique also combines loops with incompatible headers, it suffers from the same limitations as most loop transformation techniques which are based on the polyhedral model (see [18] for a description of the limitations). After every fusion step, we also reevaluate which loop pairs can be combined and re-compute the fusion gains for the newly generated loops.

As long as profitable loop pairs remain, we repeat lines 6-9. Finally, we generate the fused C-code from our internal program model (line 10). The complexity of our loop fusion algorithm is proportional with the number of times we call the MinII $(O(m * m)$ where $\mathrm{m}$ is the number of fusible loops). The greedy nature of this algorithm does not prevent us from obtaining good results (see Sect. 6).

\section{Experiments}

We quantify the performance benefits of morphing with several tasks extracted from typical multimedia applications (Tab. 1). $\mathrm{mm}$ is a matrix multiplication. fir is a finite impulse response filter. conv convolves an image with a 3x3 convolution kernel. $d c t$ and wave respectively stand for the DCT and wavelet transformation, both used in most image 

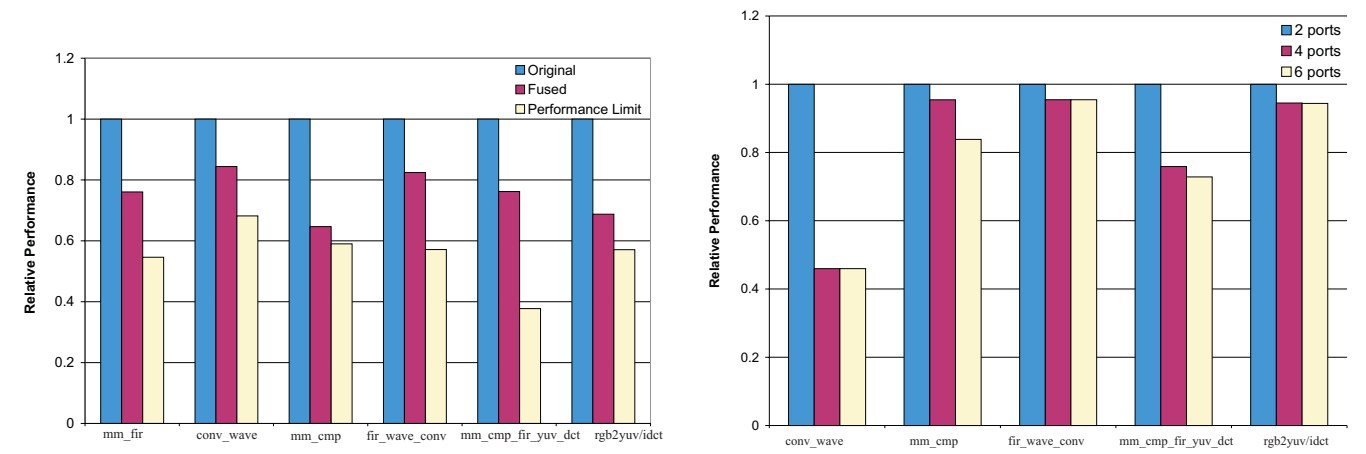

\section{Figure 5. Relative reduction in execu- Figure 6. Influence of the number of tion time memory ports on the performance}

compression algorithms. cmp compares two images, writing the maximum value of each pixel in a third matrix. Finally, rgb2yuv implements a YUV to RGB transformation of an image. Real systems (e.g., MPEG4 IM1 player and MPEG21 3D encoder) typically consist of several of these image/video processing tasks. To mimic their behavior, we combine these media benchmarks in typical tasks-sets or scenarios.

\begin{tabular}{|l|l|l|l|}
\hline application & number of loop nests & max. depth & perfectly nested \\
\hline \hline mm & 2 & 3 & no \\
\hline dct/idct & 1 & 4 & no \\
\hline rgb2yuv & 2 & 3 & no \\
\hline fir & 1 & 3 & no \\
\hline wave & 3 & 2 & no \\
\hline conv & 1 & 4 & no \\
\hline cmp & 1 & 2 & yes \\
\hline
\end{tabular}

Table 1. The benchmark applications: number of loops inside each application(2), maximum depth of a loop nest(3), (im)perfectly nested(4)

Performance numbers are obtained with a VLIW simulator (Trimaran). We simulate a non-speculative processor with four integer functional units, two floating point units and four memory ports. We have modified the compiler to make it aware of the data assignment constraints. Our compiler schedules only memory operations in parallel if their corresponding data are stored in different memories. For our experiments, we generate the results for the fastest possible assignment. In this assignment, accesses to different data can be scheduled in parallel. However, we exclude parallel accesses to the same data structure, since this would require energy-hungry multi-port memories. This assignment corresponds to a conflict graph in which edges exist between each pair of data, but no self-edges.

We first quantify the performance improvement for several scenarios (Fig. 5). As a reference, we have measured the performance of the original code (first bar). Secondly, we show the performance improvement after morphing (second bar). Finally, we indicate the execution time of the longest loop nest of each scenario (third bar). This is an upper bound of the performance improvement that we can obtain with fusion. It assumes that all tasks in the scenario can be fused with the longest one. This is only possible when sufficient resources exist on the platform to schedule all operations in parallel. However, on our platform only a limited number of memory ports, registers and functional units are available. Hence, in practice the third bar remains an unreachable upper bound for the 


\begin{tabular}{|l|l|l|l|l|}
\hline Application & $\begin{array}{l}\text { Morphing } \\
\text { steps }\end{array}$ & $\begin{array}{l}\text { Number } \\
\text { morphing } \\
\text { candidates }\end{array}$ & $\begin{array}{l}\text { Overlap after } \\
\text { morphing } \\
\text { best }\end{array}$ & $\begin{array}{l}\text { Overlap after } \\
\text { morphing } \\
\text { second best }\end{array}$ \\
\hline \hline mm_fir & 3 & 11.5 & 0.86 & 0.86 \\
\hline r2yidct & 4 & 13.5 & 1 & 1 \\
\hline conv_wave & 1 & 6 & 0.98 & 0.895 \\
\hline mm_cmp & 2 & 8.6 & 0.96 & 0.82 \\
\hline fir_conv_wave & 2 & 2.3 & 1 & 1 \\
\hline $\begin{array}{l}\text { mm_cmp_fir } \\
\text { r2y_idct }\end{array}$ & 4 & 67.5 & 0.929 & 0.966 \\
\hline
\end{tabular}

Table 2. Morphing heuristic exploration space: number of morphing decisions taken (2), average number of possible morphing candidates in each step (3), average number of iterations overlapped with our morphing heuristic compared to optimal morphing resp. for the best morphing (4) and the second best morphing candidate (5)

performance.

Morphing improves the performance for all scenarios between $10 \%$ and $38 \%$. Three factors mainly determine how much morphing improves the performance. First, how badly the original loops use the available bandwidth. Morphing adds extra memory accesses to the fused block. The compiler can use them to better fill the memory access slots. The more memory access slots are originally empty in the basic blocks, the easier fusion can fill them while keeping the original schedule length unchanged. E.g., yuv accesses many times the same array in each basic block. Since we do not use multi-port memories, the compiler has to serialize these accesses. The memory access schedule of the basic block is then long and contains many empty access slots. Hence, fusing here really helps to improve the system's performance. For rgb2yuv_dct the performance even comes close to the upper bound. Secondly, it depends on how well the initial iteration domains are balanced. If one loop dominates the execution time, fusion it with the other ones does not result in large performance gains. The dominant loop determines the execution time anyway. Thirdly, the compatibility of the initial loop headers is important. The more compatible the loop headers originally are, the fewer extra loops we need to fuse them. Hence, the less extra control overhead is added to the fused code.

Tab. 2 quantifies the design space that our iterative morphing algorithm explores. First, we indicate how many iterations it executes for each scenario (column 2). In each iteration it selects from a wide range of possible loop pairs. We show the average number of loop pairs in the third column. Traditional techniques cannot fuse any of these loop nests, because they are not conformable. Morphing thus considerably increases the applicability of fusion. Finally, we indicate how well our morphing heuristic overlaps two non-compatible iteration domains (columns 4-5). We compare the number of fused iterations after morphing of a loop pair $(11,12)$ to the maximal number of iterations that can be fused, i.e. $\mathrm{Nr}_{\text {iter. }}^{\text {morphed }}(11,12) / \min .\left(\mathrm{Nr}_{\text {iter. }}(11), \mathrm{Nr}_{\text {iter. }}(12)\right)$. The closer this number approaches one, the more efficient we can morph both iteration domains. We show this metric both for the loop pairs with the best and second highest fusion gain. In any case, it is larger than 0.82 . This is a clear indication of the effectiveness of our morphing algorithm.

In a last experiment, we quantify how adding more memory ports influences the performance. Generally, the original code does not benefit from many memory ports, because only few independent memory operations exist in each basic block. With fusion we increase 
the number of independent operations. Hence, we can fill more memory ports on the platform and decrease the execution time (see Fig. 6). For all the instances, increasing the number of memory ports results in performance gains. The performance gains depend on the application. If the available bandwidth really determines the performance (such as in of $m m_{-} c m p_{-} f r_{-} y u v_{-} d c t$ ), the more ports exist, the higher the performance gains become. In contrast, in some applications (e.g. conv_wave and fir_wave_conv), even after fusion many memory access slots remain unused. Hence, adding more memory ports does not help.

\section{Conclusion}

In this paper, we have presented loop morphing, a technique to fuse loops with unconformable loop headers. It is a combination of loop fusion with loop splitting and strip mining. With loop morphing we can optimize the memory bandwidth on VLIW across the boundaries of a basic block. Because morphing significantly increases the number of loops which can be fused, we have also introduced a technique to decide which loops to fuse first while optimizing the memory bandwidth. We have implemented our approach in SUIF and can automatically generate the morphed code. We have measured performance improvements up to $38 \%$ for realistic multi-media tasks.

\section{Acknowledgments}

This work was supported by the IWT Flanders and the Spanish Government Grant TIC $2002-0750$.

\section{References}

[1] L. Benini and G. De Micheli. System-level power optimization: techniques and tools. TODAES, 5(2):115-192, 2000.

[2] F. Bodin, et al. A quantitative algorithm for data locality optimization. In Proc. Int. Wkshp. on Code Generation, 1991.

[3] K. Danckaert et al. A preprocessing step for global loop transformations for data transfer and storage optimization. CASES 2002.

[4] D. Gannon et al. Strategies for cache and local memory management by global progra, optimizations. J. of Parallel and Distributed Systems, 25:587-617, 1988.

[5] P. Grun et al. Memory Aware Compilation through Timing Extraction. In Proc. 37th Dac 2001.

[6] Ken Kennedy and Kathryn S. McKinley. Maximizing loop parallelism and improving data locality via loop fusion and distribution. In 1993 Workshop on Languages and Compilers for Parallel Computing Portland, Ore., 1993. Berlin: Springer Verlag.

[7] K.McKinley et al. Improving data locality with loop transformations. ACM Transactions on Programming Languages and Systems, 18(4):424-453, July 1996.

[8] D. Kuck. Parallel Processing of ordinary programs. Advances in Computers, 15(4):119-179, 1976.

[9] L. Lamport. The parallel execution of do-loops. Communications of ACM, 17(2):83-93, Feb. 1974.

[10] A. W. Lim and M. S. Lam. Maximizing parallelism and minimizing synchronization with affine transforms. In Proceedings of the Twenty-fourth Annual ACM Symposium on the Principles of Programming Languages, Paris, France, 1997.

[11] M.Wolf. Improving locality and parallelism in nested loops. Technical report, Technical report CSL-TR-92-538, Stanford Univ., CA, USA, Sep. 1992.

[12] P. Panda et al. Exploiting Off-Chip Memory Access Modes in High-Level Synthesis. In Proc. Iccad, pages 333-340, Oct. 1997.

[13] A. Porterfield. Software methods for improvement of cache performance on supercomputer applications. PhD thesis, Rice University, 1989.

[14] P.Panda et al. Data and Memory Optimizations for Embedded Systems. ACM Trans. on Design Automation for Embedded Systems (TODAES), 6(2):142-206, Apr. 2001.

[15] Y. Qian et al. Loop Fusion for Clustered VLIW Architectures. In Proc. Joint Conference on Languages, Compilers and Tools for Embedded Systems and Software and Compilers for Embedded Systems, pages 19-21, June 2002.

[16] B. Rau. Iterative Modulo Scheduling. Technical report, HP Labs, 1995.

[17] M. Saghir et al. Exploiting Dual Data Banks in Digital Signal Processors. In ASPLOS, Jun. 1996.

[18] S. Verdoolaege et al. Multi-dimensional incremental loop fusion for data locality. In Proceedings 2003 Application-specific Systems, Architectures and Processors, pages 17-27, 2003.

[19] W. Verhaegh et al. A Two-stage Solution Approach for Multidimensional Periodic Scheduling. IEEE Trans. Computer Aided Design of Integrated Circuits and Systems, 10(10):1185-1199, Oct. 2001.

[20] S. Wuytack et al. Minimizing the required memory bandwidth in VLSI system realizations. IEEE Trans. VLSI Systems, 7(4):433441, Dec. 1999. 